\title{
DU COUP DE TÊTE DE ZIDANE À LA GRÈVE DE KNYSNA. FOOTBALL, SENTIMENTS ET SOCIETE DANS LA FRANCE XXI ${ }^{\mathrm{e}}$ SIECLE*
}

Da cabeçada de Zidane à greve de Knysna. Futebol, sentimentos e sociedade na França do século XXI

From Zidane's headbutt to the Knysna strike. Football, feelings and society in $21^{\text {st }}$ Century France

\author{
Michel Raspaud $^{* *}$
}

\begin{abstract}
RÉSUMÉ
A Berlin, en 2006, Zidane termine sa carrière sur un "coup de tête". Quatre ans plus tard, en Afrique du Sud, non seulement l'équipe de France de football déploie un jeu de faible qualité, mais les joueurs ont un comportement indigne, allant jusqu'à faire la grève de l'entraînement. Tous ces événements conduisent supporteurs, médias et politiques à exprimer des sentiments passionnels. Ces derniers illustrent les fondements de l'attachement à l'équipe nationale.

Mots-clés: football; équipe nationale; presse écrite, sentiments; France.

\section{RESUMO}

Em Berlim, em 2006, Zidane encerrou sua carreira com uma "cabeçada". Quatro anos mais tarde, na África do Sul, não apenas a equipe futebol da França apresenta um jogo de baixa qualidade como os jogadores têm um comportamento indigno, chegando a fazer greve de treinamento. Todos estes eventos levam torcedores, mídia e políticos a expressar sentimentos passionais. Eles ilustram os fundamentos de envolvimento em relação à equipe nacional.
\end{abstract}

Palavras-chave: futebol; equipe nacional; imprensa escrita, sentimentos; França.

\footnotetext{
Une première version de ce texte a été présentée sous forme de conférence au Département d'Histoire de l'Universidade Federal do Paraná, à Curitiba, le 15 mai 2012.

** Université Joseph Fourier, Grenoble, France.
} 


\begin{abstract}
In Berlin in 2006, Zidane ended his career on a "headbutt". Four years later, in South Africa, not only the French national team deploys a low football quality, but the players have an undignified behaviour, up to strike training. All these events lead supporters, media and politicians to express feelings of passion. They illustrate the foundations of attachment to the national team.
\end{abstract}

Key-words: football; national team; newspapers; feelings; France.

C'est au cours des toutes dernières décennies du $\mathrm{XX}^{\mathrm{e}}$ siècle que le sport a pris une place considérable dans les sociétés contemporaines, en particulier grâce à son spectacle télévisé qui permet aux supporteurs d'assister aux matches et compétitions quel que soit le lieu où ils se déroulent. Parmi l'ensemble des sports, le football occupe une position particulière puisqu'il peut être considéré comme le sport le plus populaire du monde en termes de pratique et de spectacle, le plus apprécié dans la grande majorité des pays. Aussi, les grands matches domestiques, appelés derby ou clássico, les grandes compétitions internationales entre clubs (Ligue des Champions, Copa Libertadores...), et les grands tournois qui rassemblent les équipes nationales (Coupe du Monde, Euro, Copa América...), sont des moments privilégiés pour ce qui concerne le spectacle sportif mais aussi pour l'expression des émotions et des "passions partisanes". ${ }^{1}$ A ces occasions, les supporteurs, qu'ils soient rassemblés dans les stades ou devant des écrans de télévision, vivent à l'unisson leur passion, partagent collectivement tout un ensemble d'émotions qui expriment en quelques dizaines de minutes presque toute la gamme de sentiments qu'un individu peut éprouver au cours d'une vie.

Ce dernier aspect, les émotions et sentiments éprouvés par les spectateurs au cours d'un match, a été et demeure fortement vilipendé par la "sociologie critique", voyant dans le sport en général et le football en particulier une forme d'aliénation du citoyen manipulé par le pouvoir. ${ }^{2}$ Ainsi, le spectacle du football est-il pensé par cette sociologie comme "une politique d'encadrement pulsionnel des foules, un moyen de contrôle social,

1 BROMBERGER, Christian (avec la collaboration de Alain Hayot \& Jean-Marc Mariottini). Le match de football. Ethnologie d'une passion partisane à Marseille, Naples et Turin. Paris: Editions de la Maison des Sciences de l'Homme, 1995.

2 BROHM, Jean-Marie. Sociologie politique du sport. Paris: Jean-Pierre Delarge, 1976. 
une intoxication idéologique qui sature tout l'espace public". ${ }^{3}$ Si une grande partie des critiques faites au spectacle sportif professionnel paraît dans une certaine mesure recevable (aliénation du sportif lui-même par les exigences physiques de l'entraînement et de la compétition, tricheries et manipulations récurrentes de compétitions, dopage systématisé dans certains sports, salaires extravagants des stars, ${ }^{4}$ etc.), on ne peut nier non plus que dans toute société, quelle qu'elle soit, une part de rêve, d'imaginaire, de projection doive trouver un espace de formulation au sein duquel s'expriment sentiments, émotions et passions. En cela, le sport en général, et le football en particulier, peut être qualifié de "fait social total" pour reprendre l'expression forgée par Marcel Mauss, étant traversé par l'économie, la politique, le sociétal, le religieux, l'identitaire et le communautaire, etc. Il renvoie de ce fait à tous les aspects qui touchent à l'humain en quelque sorte:

\begin{abstract}
Dans ces phénomène sociaux "totaux", comme nous proposons de les appeler, s'expriment à la fois et d'un coup toutes sortes d'institutions: religieuses, juridiques et morales - et celles-ci politiques et familiales en même temps; économiques - et celles-ci supposent des formes particulières de la production et de la consommation, ou plutôt de la prestation et de la distribution; sans compter les phénomènes esthétiques auxquels aboutissent ces faits et les phénomènes morphologiques que manifestent ces institutions. ${ }^{5}$
\end{abstract}

Aussi, malgré les critiques politiques - au sens de gestion de la Cité - qui peuvent être adressées au sport professionnel et à son spectacle mondialisé, on peut toutefois trouver en son sein un certain nombre d'aspects qui portent à réfléchir quant aux interrelations entre les institutions dont parle Marcel Mauss, en particulier en ce qui concerne politique et morale, cette dernière devant être entendue en tant que science des mours. Quels liens établir entre politique, morale et football? La sociologie critique a son

3 BROHM, Jean-Marie; PERELMAN, Marc. Le football, une peste émotionnelle. Paris: Gallimard, 2006. p. 15.

4 En Russie, le footballeur Eto'o gagne vingt millions d' $€$ par an, soit les revenus de toute un vie de quarante travailleurs Français recevant le salaire minimum!

5 MAUSS, Marcel. (1950). Sociologie et anthropologie. Paris: Presses universitaires de France, $5^{\mathrm{e}}$ édition, 1973. p. 147. 
idée là-dessus qui a été exprimée succinctement supra. Le point de vue qui sera défendu ici est qu'il s'agit de ce que l'on pourrait appeler un næud institutionnel, c'est-à-dire que des interrelations stables existent entre ces entités (politique, morale, sport...) qui permettent de prévoir et anticiper les réactions des uns et des autres acteurs impliqués dans le "fait social total" en question. Car les institutions, selon la sociologie durkheimienne, peuvent être décrites comme "des manières de faire, de sentir et de penser, "cristallisées", à peu près constantes, contraignantes et distinctives d'un groupe social donné". ${ }^{6}$

\section{Sentiments et politique}

Parmi ces manières de faire, de sentir, de penser, on peut inclure les affects au sens large (sentiments, émotions, passions...) qui sont partagés au sein d'une collectivité particulière (nation, groupe social, ethnie...) et se manifestent dans des moments particuliers, à propos de faits identifiés, et sur des modes d'expression conventionnels. Pierre Ansart souligne qu'il faut "reconnaître l'universalité des affects associés à la vie en commun et mettre en évidence les particularités de chaque situation et de chaque formation historique". 7 Il souligne que ces affectivités sont durables, non violentes, mais également peu explicitées, telles que les ressentiments. Aussi lui paraît-il important, aujourd'hui, que 'l'étude des sentiments politiques, interroge, non plus seulement les moments exceptionnels de l'histoire (révolutions, crises sociales, événements dramatiques), [mais] davantage les processus que les convulsions: l'évolution des sensibilités, l'émergence d'un charisme, la constance d'une résistance aux oppressions, les identités sociales..." (p. 11-12). Plus loin, il écrit aussi que "de même l'accent n'est pas mis sur les seules individualités, sur la personnalité de tel chef, mais sur les affectivités collectives, sur les sentiments partagés dans une cité, une ethnie, une nation.

6 BOUDON, Raymond; BOURRICAUD, François. Dictionnaire Critique de la Sociologie. Paris: Presses Universitaires de France, 1982. p 307.

7 ANSART, Pierre. Sciences sociales et Sentiments politiques. Présentation. In: HAROCHE, Claudine (Dir.). Les sentiments et le politique. Paris: L'Harmattan, 2007, p.11-19. (p. 11). 
L'attention sera portée vers les sentiments communément partagés, qui peuvent constituer une dimension, certes obscure, mais essentielle, d'une culture et dont les changements peuvent participer aux bouleversements sociaux et politiques" (p. 12). Il s'agira alors, dans le texte qui suit, d'essayer de répondre au programme proposé par Pierre Ansart, à savoir "quels sont, dans une nation, dans une aire de civilisation, les sentiments dominants et quels en sont les manifestations et les signes?" (p. 12).

Compassion, colère, peur, terreur, courage relèvent de, et incarnent des passions politiques. L'acteur social accepte des normes ou bien s'insurge, il se sent en sécurité, ou outragé, ou bien dans la détresse: "ces passions constituent des actes, à travers lesquels le regard anthropologique peut chercher à analyser les dimensions sociales et politiques des passions" ${ }^{8}$ Cependant, ces passions s'expriment par une rhétorique, des manifestations, des effets de la colère qui participent d'un "dispositif pathétique" lors de différents moments historiques mais aussi au regard des événements de l'actualité.

Les passions impliquent des identités tout autant individuelles que sociales. Ces passions se voient particulièrement mobilisées lorsque ces identités se trouvent questionnées, mises en jeu, lorsque le rapport au monde d'un groupe social, d'une société, et avant tout lorsque les conceptions morales qui sous-tendent ce rapport sont ébranlées, inquiétées dans leur intégrité, voire menacées de destruction: “c'est dans cette mesure que la menace joue un rôle dans les passions politiques, qui sont associées à la blessure, et en fin de compte articulées à un enjeu d'offense morale et politique". ${ }^{10}$ A partir de là, il s'agira de montrer comment les passions font partie d'actions et de raisons qui questionnent profondément l'ordre social constitué:

D'Hérodote à Machiavel, de Thucydide à Rousseau, les êtres humains étaient des animaux politiques - mais ils étaient politiques parce qu'ils étaient pathétiques. Ils souffraient la crainte, l'envie et la colère; ils jouissaient de la fierté, de la gloire ou de l'espoir - et ces émotions les amenaient à prendre

8 PANDOLFI, Mariella; CRAPANZANO, Vincent Crapanzano. Les passions: au cœur du politique? Anthropologie et Sociétés, v. 32, n. 3, p. 7-13, 2008. (p. 7).

9 Mariella Pandolfi \& Vincent Crapanzano, art. cit., p. 10.

10 Ibid., p. 10-11. 
des décisions et à agir, à commander ou à obéir, ou encore à s'insurger. Individus et société, souverains et multitude: les êtres humains agissent à cause des passions qui les meuvent, et qui sont, pour eux, des raisons - et des raisons pas moins vraies que la puissance ou l'argent. L'argent et la puissance, en effet, peuvent devenir vrais pour des êtres humains, seulement parce qu'ils sont ressentis dans l'humiliation et la misère; l'arrogance et le mépris; l'avidité et l'autosatisfaction. Récupérer ce sens et cette sensibilité, loin de constituer un oubli naïf du pouvoir, des causes économiques, ou de la lutte des classes, permet la redécouverte nécessaire, à ce jour pressante, d'une intelligence anthropologique. ${ }^{11}$

Pour l'auteur des lignes supra, les grands philosophes moraux considéraient les passions comme des jugements, des croyances, des pensées qui se produisaient en réaction à des situations réelles. C'est ce que ce texte essayera de montrer à travers une série d'événements singuliers, mais significatifs, survenus à l'équipe nationale de football, qui expriment la réalité concrète d'un certain nombre de valeurs profondément ancrées dans la société française.

E o futebol é, por excelência, um desses lugares em que a afetividade da política se manifesta para além da racionalidade pragmática. Fortemente contido de paixão - individual e coletiva - o futebol não se prende exclusivamente às determinações de classes, na medida em que, tomadas de forma clássica, elas são excessivamente redutoras. Nesse sentido, a dimensão afetiva da vida esportiva é também marcada pela paixão e pelo ressentimento. É, portanto, o lugar da "inconsciência", no sentido oposto daquela consciência racional determinada e imposta de cima e de fora dos sujeitos e dos grupos. É um espaço privilegiado do inconsciente coletivo, dos indivíduos e das massas. ${ }^{12}$

En effet, depuis fort longtemps se cristallise, sur l'équipe de France de football, un certain nombre de valeurs, lesquelles incarnent d'une

11 SISSA, Giulia. Passions politiques. Un défi pour l'anthropologie contemporaine. Anthropologie et Sociétés, v. 32, n. 3, p. 173-177, 2008. (p. 174).

12 RIBEIRO, Luiz Carlos Ribeiro. O futebol no campo afetivo da história. Movimento, v. 10, n. 3, p. 99-11, set./dez. 2004. (p. 106). 
certaine manière ce que la société française pense d'elle-même, la manière dont elle se représente à elle-même et celle dont elle souhaite apparaître aux yeux des autres. Ainsi, pour parler du beau jeu qu'elle doit pratiquer, parle-t-on d'un "football champagne", ${ }^{13}$ c'est-à-dire d'un football pétillant, subtil, euphorisant, procurant du plaisir aux supporteurs et aux spectateurs, football illustré principalement par la grande équipe du Stade de Reims ${ }^{14}$ au cours des années 1950, ${ }^{15}$ deux fois finaliste de la Coupe d'Europe des clubs champions ${ }^{16}$ (1956 et 1959), et base de l'équipe nationale demi-finaliste de la Coupe du Monde 1958 en Suède. Ce football s'est trouvé incarné plus tard par l'équipe de la décennie 80 d'abord, ${ }^{17}$ puis par celle des années 1998-2000 ensuite. ${ }^{18}$ Lors de chacune de ces trois périodes historiques de l'équipe nationale, un joueur reconnu de classe mondiale en était le leader technique: Raymond Kopa, Michel Platini, Zinedine Zidane. Les supporteurs vivent sur ce passé plus ou moins récent et son alors très exigeants en ce qui concerne le football produit par l'équipe nationale qui a toujours, en France, été pensée comme au-dessus de celui des clubs. ${ }^{19}$

Qui plus est, et l'on ne doit pas l'oublier, le sport français est également marqué par la figure tutélaire du baron Pierre de Coubertin, rénovateur des Jeux olympiques, aristocrate ardent défenseur de l'amateurisme contre le professionnalisme, et dont l'un des principes était la participation et le fair play plus que la victoire ("l'important, c'est de participer"!). C'est dans ce contexte historique qu'il faut comprendre les faits suivants et les réactions passionnelles qu'ils ont produites.

13 En France, on parle de football champagne comme au Brésil on parle de futebol arte.

14 On comprend l'allusion lorsque l'on sait que Reims est la capitale de la région qui produit le Champagne. Dans le même temps, le Champagne s'inscrit dans une série de produits "de luxe" qui illustrent, tous ensemble, un certain art de vivre "à la française" (vins fins, gastronomie, parfums, haute couture, littérature, savoir vivre et politesse...) caractéristique de la nation et de la représentation qu'elle se fait d'elle-même.

15 Cinq fois Champion de France en dix ans (1953, 1955, 1958, 1960, 1962).

16 Ancienne appellation de la Ligue des champions (Champions League), équivalent de la Copa Libertadores.

17 Demi-finaliste de la Coupe du monde en 1982 (Espagne) et 1986 (Mexique), championne d'Europe en 1984 (France).

18 Championne du monde en 1998 (France), puis d'Europe en 2000 (Belgique - Pays-Bas).

19 Cf. la thèse de doctorat en sociologie de Hassen Slimani, La professionnalisation du football français: un modèle de dénégations. Nantes: Université de Nantes, 30 novembre 2000. 


\section{Football et passions françaises 1: le coup de tête de Zidane}

En juillet 2006, lors de la finale de la Coupe du Monde de football à Berlin entre la France et l'Italie, à la $107^{\mathrm{e}}$ minute, durant les prolongations, Zinedine Zidane donne un coup de tête au joueur de la Squadra Azzura, Materazzi. C'est le dernier match de sa carrière de footballeur professionnel, laquelle aurait pu se terminer en apothéose: par la victoire finale et un second titre de champion du monde. Au lieu de cela, c'est l'expulsion du terrain après que le juge de touche ait informé l'arbitre central du mauvais geste de Zidane. La France perd le match lors de la séance des tirs au but.

Ce geste de Zidane ne sera toutefois pas vraiment condamné par les commentateurs sportifs, les journalistes, les supporteurs et l'ensemble de la population. Certes, ce n'est pas la règle de se faire justice soi-même (on peut supposer que si Zidane a fait un tel geste, spectaculaire et "efficace", c'est qu'il y avait un lourd contentieux physique et/ou moral entre les deux joueurs), mais l'auteur de ce "mauvais geste" avait tellement apporté au football français (Coupe du Monde 1998) et international (Juventus de Turin, Real Madrid), il était une telle star mondiale (désigné la veille même de la finale comme le meilleur joueur du tournoi), que toutes sortes d'excuses pouvaient être mobilisées pour absoudre Zidane. ${ }^{20}$

\section{Football et passions françaises 2: la main de Thierry Henry}

Al'automne 2009, la France doit passer par deux matches de barrage contre la République d'Irlande pour se qualifier pour la Coupe du Monde 2010 en Afrique du Sud. Si elle gagne le premier match à Dublin (1-0), lors du match retour, au Stade de France, elle est menée 1 but à zéro dans la

20 DELBÉE, Anne. La $107^{e}$ minute. Paris: Les Quatre Chemins, 2006; DURET, Pascal. Perception du "coup de boule" de Zidane par les collégiens. Socio-logos. Revue de l'Association Française de Sociologie [en ligne], 2/2007. 
prolongation, avant que William Gallas ne marque le but de la qualification. A la $103^{\mathrm{e}}$ minute, lors d'une action de jeu sur le coté gauche, à proximité des buts irlandais, Thierry Henry contrôle le ballon de la main pour le ramener sur son pied et le transmettre à Gallas, qui marque. Apparemment, seuls les arbitres n'ont pas vu le contrôle de la main de Thierry Henry.

Aussitôt, malgré le gain de la qualification, tout un ensemble de polémiques passionnelles émergent dont se font l'écho les commentateurs sportifs, mais pas seulement. En particulier, la joie du sélectionneur et de l'ensemble du staff technique après le but de Gallas suite à l'action frauduleuse de Thierry Henry, paraît déplacée pour ceux-là (il faut déjà souligner que le sélectionneur est critiqué par la presse depuis le catastrophique Euro $2008^{21}$ ). Pourtant, personne ne s'est posé la question suivante: si l'arbitre, à quelques mètres de l'action, n'a pas vu la main de Thierry Henry, comment le sélectionneur et le staff technique auraient-ils pu la voir, alors qu'il se trouvaient à cinquante mètres et au ras du sol? On oublie que téléspectateurs et journalistes sont dans des positions spatiales privilégiées par rapport à l'arbitre, et qu'ils disposent du replay de la télévision.

Des questions morales ont alors surgi:

- Peut-on aller en Afrique du Sud après un tel but?

- Ne faut-il pas rejouer le match?

- Pourquoi Thierry Henry ne s'est-il pas dénoncé auprès de l'arbitre (!), lui indiquant qu'il avait contrôlé le ballon avec la main?

- Comment le sélectionneur, le staff technique, et les dirigeants pouvaient-ils exprimer une telle joie, après un tel but?

Enfin, des questions que l'on se pose lorsque l'on a la chance d'être riche et bien portant! Moins quand on est une équipe moribonde qui ne parvient pas à aligner deux matches consécutifs de qualité acceptable. Et surtout, lorsque l'on est un joueur de football professionnel, un sélectionneur national, ou un président de fédération qui connait les enjeux d'une telle qualification: émotionnels, sportifs, de prestige, certes, mais aussi et principalement financiers (droits télévisuels, partenariats commerciaux, marchandisage, etc.).

21 Championnat d'Europe des nations en juin 2008, organisé en Suisse et en Autriche: élimination sans gloire après un match nul et deux défaites. En direct à la télévision, le sélectionneur laisse alors entendre que cela n'a pas vraiment d'importance, et profite de l'antenne pour faire publiquement sa demande en mariage à sa compagne, Estelle Denis, animatrice d'émissions télévisées! 


\section{Football et passions françaises 3: la "grève" de Knysna 22}

Lors de la Coupe du Monde en Afrique du Sud en 2010, les choses vont empirer pour les joueurs de l'équipe de France bien qu'ils ne soient alors les auteurs d'aucun mauvais geste lors des matches de cette compétition. Car cette fois, commentateurs sportifs, journalistes, supporteurs, et même jusqu'au personnel politique fustigeront unanimement le comportement des footballeurs français rassemblés en Afrique du Sud: courte généalogie.

En effet, entre 2006 et 2010, et bien que le sélectionneur de l'équipe nationale soit demeuré le même, les résultats sont décevants: ${ }^{23}$

- Lors de l'Euro 2008, en Suisse et Autriche, la France fait match nul contre la Roumanie (0-0), puis est sévèrement battue par les Pays-Bas (1-4) et l'Italie (0-2).

-A l'automne 2009, la France ne doit sa qualification pour l'Afrique du Sud en éliminant la République d'Irlande, que par une action de but entachée d'une main que seuls les arbitres n'ont pas vue.

- Enfin, lors de cette Coupe du Monde 2010, la France fait match nul contre l'Uruguay (0-0), avant de perdre sans gloire contre le Mexique (0-2), puis contre l'Afrique du Sud (1-2).

Ce que l'on va traiter ici, ce ne sont pas les piètres résultats sportifs de l'équipe nationale (pour qu'il y ait un vainqueur, il faut bien qu'il y ait des vaincus), mais la série des événements qui se sont déroulés dans le cadre de cette équipe, et les conséquences en termes de commentaires faits sur ces événements, lesquels (événements et commentaires) renvoient à la thématique des sentiments qui peuvent relier un "peuple", en l'occurrence les Français, à "son" équipe nationale de football. Car en effet, au-delà des résultats décevants, un certain nombre d'événements, de faits, d'actions

22 Knysna é a região da África do Sul onde se concentrou o selecionado francês, durante a Copa do Mundo de Futebol, em 2010. [Nota do Editor]

23 A la décharge du sélectionneur, les "cadres" (Zidane, Thuram et Makélélé) avaient été rappelés durant l'été 2005 pour sauver l'équipe nationale d'une non qualification, et surtout les finances de la fédération qui étaient en très mauvais état, et que la non qualification pour la Coupe du monde aurait encore aggravées. 
inimaginables ou impensables (car n'étant encore jamais advenus), ont eu lieu qui permettent alors de mieux saisir les rapports qui existent entre la foule des supporteurs, le peuple, et le représentant symbolique de celui-ci que constitue une équipe sportive nationale.

C'est donc dans les circonstances décrites supra que l'équipe nationale se retrouve en Afrique du Sud en juin 2010. Auparavant, toutefois, d'autres éléments du contexte purement sportif ${ }^{24}$ doivent encore être précisés:

1 - quelques semaines avant la Coupe du Monde, trois joueurs (Ribéry, Govou, Benzema $)^{25}$ sont interrogés par la police dans une affaire de mœurs: quelques mois auparavant, ils ont utilisé les services d'une prostituée, Zahia, alors que la jeune femme était encore mineure au moment des faits;

2 - lors de la préparation de la compétition, les joueurs affirment aux médias qu'ils vont en Afrique du Sud pour gagner la Coupe du Monde, ${ }^{26}$

3 - toutefois, il est apparent qu'existent des dissensions entre joueurs au sein de cette équipe, et que le sélectionneur ne semble pas être capable de les faire disparaître;

4 - les matches préparatoires à la compétition sont une nouvelle fois décevants, les résultats empirant au fil du temps: après un victoire contre le Costa Rica (2-1) le 26 mai, les Bleus font match nul contre la Tunisie (1-1) le 30 mai, avant d'être battus (0-1) le 4 juin par l'équipe de la République Populaire de Chine ${ }^{27}$ lors d'un match dans l'île de La Réunion; 5 - enfin, Madame Rama Yade, la Secrétaire d'Etat au Sport, trouve scandaleux que l'équipe nationale soit installée dans un palace au prix de journée astronomique, alors qu'autour sévit la pauvreté. Bien que Secrétaire d'Etat au Sport, elle ne savait pas que c'est la FIFA qui prend en charge l'hébergement des équipes participant au tournoi!

24 Nous laissons de côté les polémiques initiées par la presse sportive relatives aux primes touchées par les différents membres du staff technique et les joueurs sélectionnés durant la phase de qualification (août 2008 - novembre 2009): $236000 €$ pour un joueur n'ayant été appelé qu'une seule fois, 862000 $€$ pour le sélectionneur national! Bien entendu, cet aspect financier a pesé moralement sur le contexte.

25 Si Franck Ribéry (Bayern Munich) et Sydney Govou (Olympique Lyonnais) seront retenus parmi les 23 joueurs pour l'Afrique du Sud, tel ne sera pas le cas pour Karim Benzema (Real Madrid).

26 "On vient ici pour aller au bout", déclare Patrice Evra, le capitaine de l'équipe nationale (Le Figaro, 11 juin 2010, p. 4).

27 Classée au $84^{\mathrm{e}}$ rang mondial par la FIFA à cette date! 
Cependant, plus que les médiocres performances de l'équipe (0-0 contre l'Uruguay, puis 0-2 contre le Mexique), l'événement qui a déclenché le scandale est le suivant: dans les vestiaires, durant la mi-temps du match France - Mexique, le sélectionneur fait une réflexion à Nicolas Anelka sur son jeu, car celui-ci ne respecte pas ses consignes. En réponse, le joueur marmonne et l'insulte. En conséquence du non respect des consignes, il se voit remplacé et ne reprend pas le match.

Le samedi 21 juin, après quarante-huit heures d'enquête, le journal L'Equipe fait paraître en Une et en gros caractères, les mots que Nicolas Anelka aurait prononcés à l'encontre du sélectionneur. Les dirigeants de la Fédération française de football apprennent ainsi par la presse, l'incident et l'altercation entre Domenech et Anelka. Il est demandé à ce dernier de s'excuser publiquement (devant les médias), ce qu'il refuse de faire. Il est alors exclu du groupe et rentre en Angleterre (il jouait à Londres pour le club de Chelsea).

Le lendemain, arrivant sur le terrain d'entraînement de Knysna, les joueurs refusent de sortir du bus de l'équipe nationale et de s'entraîner. Ils ont, entre temps, rédigé une déclaration que le sélectionneur, Raymond Domenech, se voit obliger de lire devant les micros des radios et des télévisions, déclaration par laquelle les joueurs dénoncent les conditions dans lesquelles Nicolas Anelka a été exclu, et le fait qu'un incident de vestiaire ait été porté à la connaissance de tous par la presse.

Aussi, encore plus que les mots d'Anelka et son exclusion (le joueur avait déjà mauvaise réputation, et la presse l'a immédiatement vilipendé, rappelant ses antécédents de comportement), c'est cette attitude collective des joueurs et le refus de s'entraîner à deux jours du dernier match contre l'Afrique du Sud (qui laissait un ultime mais très faible espoir de qualification), qui ont été jugés scandaleux, et qui ont conduit à l'expression de tout un ensemble de sentiments relatifs à l'équipe nationale. C'est ce que l'on va essayer d'analyser, à travers la presse écrite et les commentaires qui ont été faits. 


\section{Football et passions françaises 4: qu'est-ce que nous dit Knysna de la société?}

Dans un premier temps, il faut signaler que les événements de Knysna et de l'Afrique du Sud n'ont pas été qu'un feu de paille, mais un véritable incendie. En effet, dès le mois suivant, et ce jusqu'en mars 2011, c'est-à-dire durant neuf mois, tout un ensemble de livres et d'études ont été publiés (onze exactement, dont dix de juillet à octobre, soit quatre mois), comme le montre le tableau suivant.

TABLEAU 1 - Livres publiés depuis les événements de Knysna.

\begin{tabular}{|c|c|c|c|c|}
\hline Auteurs & Titres & Editeurs & Pages & Dates \\
\hline Pierre Menès & $\begin{array}{l}\text { Carton rouge pour les } \\
\text { Bleus }\end{array}$ & Ed. du Rocher & 204 & Juillet 2010 \\
\hline $\begin{array}{l}\text { Eugène Saccomano } \\
\text { Gilles Verdez }\end{array}$ & Le Roman noir des Bleus & La Martinière & 153 & Juillet 2010 \\
\hline Jean-Michel Larqué & Les secrets d'un fiasco & Ed. du Toucan & 91 & Juillet 2010 \\
\hline $\begin{array}{l}\text { "Les Amoureux de } \\
\text { l'équipe de France" }\end{array}$ & $\begin{array}{l}\text { Plus jamais ça! L'échec } \\
\text { des Bleus }\end{array}$ & Hugo \& Cie & 143 & Juillet 2010 \\
\hline Jean-Pierre Paclet & L'implosion & Michel Lafon & 200 & Août 2010 \\
\hline Patrick Mignon & $\begin{array}{l}\text { Le psychodrame du football } \\
\text { français }\end{array}$ & Esprit, $\mathrm{n}^{\circ} 367$ & 18 & $\begin{array}{l}\text { Août - sept } \\
2010\end{array}$ \\
\hline Vincent Duluc & Le Livre noir des Bleus & Robert Laffont & 267 & $\begin{array}{l}\text { Septembre } \\
2010\end{array}$ \\
\hline $\begin{array}{l}\text { Damien Degorre } \\
\text { Raphaël Raymond }\end{array}$ & Histoire d'un scoop & L'Equipe & 165 & $\begin{array}{l}\text { Octobre } \\
2010\end{array}$ \\
\hline $\begin{array}{l}\text { Antoine Grynbaum } \\
\text { Romain Schneider }\end{array}$ & $\begin{array}{l}\text { Football et politique. Les } \\
\text { liaisons dangereuses }\end{array}$ & J.C. Gausewitch & 318 & $\begin{array}{l}\text { Octobre } \\
2010\end{array}$ \\
\hline Collectif & $\begin{array}{l}101 \text { propositions pour le } \\
\text { football français }\end{array}$ & L'Equipe & 223 & $\begin{array}{l}\text { Octobre } \\
2010\end{array}$ \\
\hline Stéphane Beaud & Traîtres à la nation? & La Découverte & 287 & Mars 2011 \\
\hline
\end{tabular}

A la lecture des titres des ouvrages ou études, on perçoit une certaine distance à l'événement, en restant dans le domaine du football ou de la métaphore sportive (carton rouge, fiasco, échec), en faisant appel au roman policier ou d'espionnage (roman noir, secrets), ou encore à des aspects psychologiques (implosion, psychodrame, liaisons), etc. Un seul ouvrage va plus loin, celui du sociologue Stéphane Beaud qui titre "Traîtres 
à la nations", en insistant sur les aspects relatifs au monde socioculturels et à l'immigration.

En tout état de cause, l'étude se focalisera sur les événements, et sur ce qui en a été dit sur le moment, et non pas quelques semaines ou mois plus tard, où les paroles sont devenues plus mesurées dans le cadre de supports différents (le livre, et non pas la presse quotidienne), pour essayer d'identifier les sentiments qui ont pu être mis en avant lors de ce "psychodrame du football français" pour reprendre le titre de l'étude de Patrick Mignon. C'est sur cette base qu'est proposée l'analyse qui suit, à la différence près que, dans le cas présent, l'étude se focalise bien sur une série d'événements, ceux de Knysna, et non dans le cadre d'un processus long. Quoique l'on puisse supposer, comme cela a été vu, qu'un long processus de cristallisation a eu lieu conduisant aux réactions publiques en ce qui concerne les sentiments mobilisés à propos des joueurs de l'équipe nationale.

Si l'on reprend le sens du mot sentiment dans la langue française, plusieurs aspects intéressants émergent:

- Dès l'ancien français, le mot désigne (vers 1279) un état qui relève de l'affectivité et, spécialement, une tendance affective stable et durable, d'où l'emploi également courant (au XIII e siècle) à propos de l'inclination d'une personne pour une autre, qu'il s'agisse d'amour ou d'amitié.

- Il se dit (vers 1580) d'une opinion fondée sur une appréciation subjective et non sur un raisonnement logique. ${ }^{28}$

Le tableau suivant rapporte, de façon synthétique mais non pas de manière exhaustive, car toute la presse nationale et régionale n'ont pas été dépouillé sur l'ensemble de la période, mais seulement quelques quotidiens. ${ }^{29}$

28 Dictionnaire Historique de la Langue Française, sous la direction d'Alain Rey (Paris: Le Robert, 1998, tome 3, p. 3465).

29 Les journaux suivants ont été dépouillés: Le Dauphiné Libéré (Grenoble), Aujourd'hui en France (Paris), France Soir (Paris), Le Figaro (Paris), Le Monde (Paris), Libération (Paris). Tous ces quotidiens sont généralistes. Le journal L'Equipe, seul quotidien sportif français, n'a pas été utilisé car il est à l'origine du scoop concernant les mots adressés par Anelka à Domenech, et qu'il est partie prenante du "champ" sportif (pour reprendre le concept de Pierre Bourdieu) et, en tant que tel, un acteur qui y a des intérêts. De plus, l'ancien sélectionneur Aimé Jacquet, champion du monde en 1998, eut à souffrir durant tout son temps à la tête de l'équipe nationale (1994-1998) des critiques émises par le journal à son encontre, et il voue à ce dernier une haine tenace (cf. JACQUET, Aimé. Ma vie pour une étoile. Paris: Pocket, 2000. p. 287-300; GARCIA, David. La face cachée de L'Equipe. Paris: Editions Danger Public, 2008. p. 117-233). 
TABLEAU 2 - Titres des journaux du 19 au 23 juin 2010 et les événements de Knysna.

\begin{tabular}{|c|c|c|c|c|}
\hline Titre & Journal & Date & Page & Précisions \\
\hline Ils nous ont trompés & Le Dauphiné libéré & 19 juin & p. 30 & $\begin{array}{l}\text { Après France - } \\
\text { Mexique }(0-2) \\
\end{array}$ \\
\hline \begin{tabular}{|l|} 
Ras les Bleus \\
(arrogance, individualisme...)
\end{tabular} & Libération & \begin{tabular}{|l|}
$19-20$ \\
juin
\end{tabular} & Une & \\
\hline $\begin{array}{l}\text { Des Bleus défaits, humiliés, } \\
\text { vilipendés }\end{array}$ & Le Monde & 19 juin & Une & \\
\hline Le diable, c'est Anelka & Le Dauphiné libéré & 20 juin & p. 33 & $\begin{array}{l}\text { Après le scoop } \\
\text { de L'Equipe }\end{array}$ \\
\hline $\begin{array}{l}\text { Anelka, l'injure de trop à } \\
\text { l'image des Bleus }\end{array}$ & $\begin{array}{l}\text { Aujourd'hui en } \\
\text { France }\end{array}$ & 20 juin & p. 2-3 & \\
\hline $\begin{array}{l}\text { Coup de force surréaliste des } \\
\text { Bleus qui font "grève"... } \\
\text { Jusqu'où iront-ils? }\end{array}$ & Le Dauphiné libéré & 21 juin & Une & $\begin{array}{l}\text { Après la } \\
\text { "grève" de } \\
\text { l'entraînement }\end{array}$ \\
\hline Hallucinant & Le Dauphiné libéré & 21 juin & p. 1 sport & \\
\hline Le théâtre des guignols & Le Dauphiné libéré & 21 juin & p. 2 sport & \\
\hline La mutinerie & $\begin{array}{l}\text { Aujourd'hui en } \\
\text { France }\end{array}$ & 21 juin & Une & \\
\hline $\begin{array}{l}\text { Cette équipe de France nous } \\
\text { fait vraiment honte }\end{array}$ & $\begin{array}{l}\text { Aujourd'hui en } \\
\text { France }\end{array}$ & 21 juin & p. $2-3$ & \\
\hline $\begin{array}{l}\text { Nous sommes la risée du } \\
\text { monde sportif }\end{array}$ & $\begin{array}{l}\text { Aujourd'hui en } \\
\text { France }\end{array}$ & 21 juin & p. 2 & \\
\hline Ce feuilleton n'est pas digne & $\begin{array}{l}\text { Aujourd'hui en } \\
\text { France }\end{array}$ & 21 juin & p. 4 & $\begin{array}{l}\text { Noël Le Graët } \\
\text { (ex-président de } \\
\text { la LFP) }\end{array}$ \\
\hline \begin{tabular}{|l|} 
Dramatique et pitoyable \\
\end{tabular} & $\begin{array}{l}\text { Aujourd'hui en } \\
\text { France }\end{array}$ & 21 juin & p. 5 & $\begin{array}{l}\text { Jean-François } \\
\text { Lamour, ancien } \\
\text { ministre des } \\
\text { Sports } \\
\end{array}$ \\
\hline Déserteurs & France Soir & 21 juin & Une & \\
\hline \begin{tabular}{|l|} 
Honte \\
\end{tabular} & France Soir & 21 juin & Une & \\
\hline $\begin{array}{l}\text { Le footoir. Insultes, démis- } \\
\text { sion, grève des joueurs: } \\
\text { les Bleus sombrent dans le } \\
\text { ridicule }\end{array}$ & Libération & 21 juin & Une & \\
\hline Un désastre ou un miracle? & Le Dauphiné libéré & 22 juin & Une & $\begin{array}{l}\text { Avant France - } \\
\text { Afrique du Sud }\end{array}$ \\
\hline Cachez ces Bleus & Le Dauphiné libéré & 22 juin & p. 29 & \\
\hline Un peu de dignité & $\begin{array}{l}\text { Aujourd'hui en } \\
\text { France }\end{array}$ & 22 juin & Une & \\
\hline
\end{tabular}


TABLEAU 2 - Continuation

\begin{tabular}{|c|c|c|c|c|}
\hline $\begin{array}{l}\text { La priorité, c'est la } \\
\text { reconquête de l'honneur }\end{array}$ & $\begin{array}{l}\text { Aujourd'hui en } \\
\text { France }\end{array}$ & 22 juin & p.2 & \begin{tabular}{|l|} 
Eric Besson, \\
ministre de \\
l'Identité \\
Nationale (sic) \\
\end{tabular} \\
\hline $\begin{array}{l}\text { Les Bleus visent une } \\
\text { impossible réhabilitation }\end{array}$ & Le Figaro & 22 juin & p.12 & \\
\hline $\begin{array}{l}\text { Les Bleus méritent peut-être } \\
\text { qu'on leur crache dessus }\end{array}$ & Le Figaro & 22 juin & p.12 & $\begin{array}{l}\text { Titre de } \\
\text { l'interview de } \\
\text { Zidane } \\
\end{array}$ \\
\hline $\begin{array}{l}\text { On est en train de leur cracher } \\
\text { dessus comme jamais. } \\
\text { Peut-être qu'ils le méritent }\end{array}$ & Le Figaro & 22 juin & p.12 & $\begin{array}{l}\text { Déclaration } \\
\text { réelle de Zidane } \\
\text { dans l'interview } \\
\end{array}$ \\
\hline Le retour des zéros & Le Dauphiné libéré & 23 juin & Une & $\begin{array}{l}\text { Après France - } \\
\text { Afrique du Sud } \\
(1-2)\end{array}$ \\
\hline $\begin{array}{l}\text { La France n'est pas près de } \\
\text { leur pardonner }\end{array}$ & $\begin{array}{l}\text { Aujourd'hui en } \\
\text { France }\end{array}$ & 23 juin & p. 2 & \\
\hline La France est ridiculisée & France Soir & 23 juin & p.3 & $\begin{array}{l}\text { Alain Minc, } \\
\text { économiste }\end{array}$ \\
\hline $\begin{array}{l}\text { Les "rebelles" doivent laisser } \\
\text { la place }\end{array}$ & France Soir & 23 juin & p. 5 & \\
\hline $\begin{array}{l}\text { Après le désastre, tout est à } \\
\text { reconstruire chez les Bleus }\end{array}$ & Le Figaro & 23 juin & Une & \\
\hline $\begin{array}{l}\begin{array}{l}\text { Le cauchemar est enfin } \\
\text { terminé }\end{array} \\
\end{array}$ & Le Figaro & 23 juin & p. 12 & \\
\hline Et 1 , et 2 , et 23 zéros & Libération & 23 juin & & \\
\hline
\end{tabular}

Lorsque l'on analyse l'ensemble des thèmes "sentimentaux" et "passionnels" issus des titres de la presse écrite, on peut procéder au regroupement en six catégories comme le montre le tableau suivant.

TABLEAU 3 - Les événements de Knysna et les sentiments.

\begin{tabular}{|c|l|}
\hline Sentiments & \multicolumn{1}{c|}{ Items } \\
\hline Irréalité & Hallucinant, cauchemar, surréaliste, jusqu'où iront-ils? \\
\hline Jacquerie & Mutinerie, rebelles, grève, footoir [“foutoir”] \\
\hline Néant, vide & Tromperie, pitoyable, désastre, le retour des zéros [“le retour des héros"] \\
\hline Dérision & Risée, ridicule, guignols \\
\hline Déshonneur & $\begin{array}{l}\text { Honte, reconquérir l'honneur, impossible réhabilitation, la France pas } \\
\text { près de leur pardonner }\end{array}$ \\
\hline Indignité & $\begin{array}{l}\text { Pas digne, un peu de dignité, cachez ces Bleus [“cachez ce sein que je } \\
\text { ne saurais voir”, Molière, Tartuffe], déserteurs }\end{array}$ \\
\hline
\end{tabular}


L'irréalité dans laquelle se trouve l'observateur face aux événements: hallucinant, cauchemar, surréaliste, jusqu'où iront-ils?

La jacquerie pour signifier les comportements: mutinerie, rebelles, grève, footoir ("foutoir": jeu de mots intraduisible qui joue sur la phonétique identique),

Le néant, le vide quant au jeu déployé: tromperie, pitoyable, désastre, le retour des zéros ("le retour des héros": ici aussi un jeu de mots, sauf que héros se prononce avec un " $h$ " aspiré, la liaison phonétique entre le "s" et le "h" étant faite par ceux qui parlent mal la langue) [cela renvoie aussi au chant des supporters après la victoire contre le Brésil en 1998: "Et 1, et 2, et 3-0"].

La dérision pour parler des joueurs: risée, ridicule, guignols.

Mais il semble que les deux catégories suivantes vont plus loin en ce qui concerne le rapport du peuple et des supporteurs aux joueurs et à ce qu'ils représentent:

L'honneur bafoué: honte, reconquérir l'honneur, impossible réhabilitation, la France pas près de leur pardonner.

L'indignité: pas digne, un peu de dignité, cachez ces Bleus ("cachez ce sein que je ne saurais voir", Molière, Tartuffe), déserteurs.

L'analyse se focalisera plus particulièrement sur ces deux derniers aspects.

D'après l'anthropologue Julian Pitt-Rivers, le mot "honneur" vient du latin Honos, nom d'un dieu de la guerre qui donnait aux militaires le courage de se battre. ${ }^{30}$ Pour le Dictionnaire historique de la langue française, le terme honneur renvoie, dès la seconde moitié du $\mathrm{X}^{\mathrm{e}}$ siècle au sens général de "marque de vénération, de considération (attachée à la vertu, au mérite, etc.)". ${ }^{31}$ Dès le milieu du XI $\mathrm{XI}^{\mathrm{e}}$ siècle, honneur est attesté au sens de "considération, estime dont on jouit", et est employé (1080) pour "bien

30 PITT-RIVERS, Julian. L'honneur. In: .Anthropologie de l'honneur. Paris: Hachette, 1997. p. I-XIV. (p. I). (1 ìre édition: The fate of Sechem or the Politics of Sex. Essays in the anthropology of the Mediterranean. Cambridge: Cambridge University Press, 1977).

31 Dictionnaire Historique de la Langue Française, op. cit., tome 2, p. 1.735. 
moral, sentiment que l'on a de sa dignité". En emploi absolu, honneur (1080) se dit du sentiment qui pousse à obtenir le bien moral qu'est l'honneur. ${ }^{32}$

On voit ici que les footballeurs français n'ont pas eu le courage de se battre, et en conséquence, ils ont été traités de "déserteurs". Ils ont perdu leur honneur (la considération, l'estime dont ils jouissaient); ils sont alors devenus indignes. Pour Julian Pitt-Rivers, la question de l'honneur est fondamentale dans la société méditerranéenne, et la France comme l'Espagne, les pays bordant la rive Nord de la Méditerranée, appartiennent à cette civilisation qui met en scène l'honneur à un point très élevé (en France, le duel pour des questions d'honneur, s'est prolongé très tardivement).

Pour l'anthropologue, "comme valeur morale, chacun veut se l'attribuer [1'honneur] et le nier à beaucoup de ses voisins. Devenu un objet de discussion où les individus se disputent sa possession, il donne lieu aussi à des idées et des théories qui doivent gouverner son attribution ou sa perte". ${ }^{33}$ Justement, à travers le tableau n ${ }^{0} 3$, et la mise en catégories des items identifiés, on voit très bien le processus mis en place: un comportement indigne sur le terrain et en dehors de la part des footballeurs (les Bleus, couleur du maillot des équipes nationales, frappé du coq gaulois, emblème de la nation française, à l'emplacement du cœur), ce qui conduit la presse à mettre à distance et à rabaisser les joueurs en question. Il s'agit ici d'un processus tout à fait commun bien identifié par les ethnologues ou les anthropologues, comme Claude Lévi-Strauss par exemple:

L'humanité cesse aux frontières de la tribu, du groupe linguistique, parfois même du village; à tel point qu'un grand nombre de populations dites primitives se désignent d'un nom qui signifie les "hommes" (ou parfois - dirons-nous avec plus de discrétion - les "bons", les "excellents", les “complets"), impliquant ainsi que les autres tribus, groupes ou villages ne participent pas des vertus $^{34}$ - ou même de la nature - humaines, mais sont tout au plus composés de "mauvais", de "méchants", de "singes de terre" ou d'“œufs de pou". ${ }^{35}$

UNESCO, 1961). p. 21. 
On voit ici que sur cette base des vertus, les footballeurs sont renvoyés à un espace qui n'appartient pas à la nation de laquelle ils se réclament de par leur sélection, et de par le maillot et l'emblème qu'ils portent. Julian Pitt-Rivers (1997, p. V) est encore plus précis, en ce qui concerne l'honneur:

Les bases de l'honneur collectif sont considérables: au-delà de la famille, du lignage, du pueblo [...], on les trouve dans la nation, le régiment, $n$ 'importe quelle unité à laquelle on s'est identifie, ${ }^{36}$ n'importe quelle personne à qui on s'est attachée, avec ou sans lien juridique, n'importe quelle cause religieuse, politique, morale; dans tous les cas, l'honneur est toujours ressenti comme une partie intime de son être par chacun. ${ }^{37}$

Dignité (v. 1155) est emprunté au dérivé latin dignitas "fait de mériter, mérite", également employé pour désigner les qualités qui font qu'on est digne (estime, considération, prestige).

Indigne (v. 1200) est emprunté au latin indignus "qui ne mérite pas", "qu'on ne mérite pas", "qui ne convient pas". [...] L'usage moderne (à partir du XVIe siècle) l'entend [...], absolument, d'une chose infamante (av. 1589), ou d'une personne méprisable (1665).

(Dictionnaire Historique de la Langue Française, tome 1, p. 1.085).

On constate, ici, que les footballeurs français deviennent aux yeux de leurs concitoyens, des personnes infâmes et méprisables. Ils ont perdu leur honneur en tant que déserteurs, et se retrouvent ainsi ne pas mériter de représenter la nation. Ils abaissent celle-ci en devenant la risée du monde sportif, en étant ridicules, en se comportant comme des guignols.

Il faut savoir que, dans la culture populaire française, au milieu du XIX ${ }^{\mathrm{e}}$ siècle (1856), un guignol ${ }^{38}$ est une "marionnette sans fil", et que depuis le terme s'emploie par analogie pour désigner une personne involontairement comique ou ridicule. ${ }^{39}$ D'ailleurs, depuis environ une vingtaine

36 C'est encore moi qui souligne.

37 Julian Pitt-Rivers, op. cit., p. 5.

38 Personagem do Teatro de Fantoches (BURTIN-VINHOLES, S. Dicionário de Francês. São Paulo: Globo, 36. ed. 1995. p. 247).

39 Un "guignol" est l'emploi comme nom commun (1847) du nom du canut lyonnais Guignol, dont Mourguet fait le héros de son théâtre de marionnettes, à la fin du XVIII ${ }^{\mathrm{e}}$ siècle à Lyon. (Dictionnaire Historique de la Langue Française, tome 2, p. 1.665). 
d'années maintenant, la chaîne de télévision cryptée et à péage, Canal +, a développé une émission burlesque intitulée "Les guignols de l'info", tous les soirs vers $19 \mathrm{~h} 00$, qui reprend sous forme caricaturale et comique, des marionnettes à l'effigie des personnages qui peuplent l'actualité: hommes politiques, sportifs de tout poil et spécialement les footballeurs (Ribéry, Domenech, etc.), vedettes du cinéma ou de la chanson, animateurs de télévision, etc., pour se moquer de leurs actions, leurs tics ou petites phrases...

Même si nous faisons un grand écart théorique entre l'anthropologie de l'altérité, comme on pourrait le dire à propos de Claude Lévi-Strauss, ou l'anthropologie de l'honneur, à propos de Julian Pitt-Rivers, les analyses du sociologue américain Erving Goffman présentent aussi certains intérêts pour comprendre les phénomènes identifiés supra. Goffman a proposé un certain nombre de réflexions relatives aux stigmates attachés à la condition physique ou sociale des individus dans la société, en quelque sorte leur catégorisation sociale, et à la relation dialectique qui existe avec celui qui porte le regard qui stigmatise (relation qui va dans les deux sens).

La société établit des procédés servant à répartir en catégories les personnes et les contingents d'attributs qu'elle estime ordinaires et naturels chez les membres de chacune de ces catégories. Les cadres sociaux établissent les catégories de personnes qu'il est probable d'y rencontrer La routine des rapports sociaux dans les cadres établis nous permet d'avoir affaire aux autres, habituellement présents, sans leur accorder une attention ou des pensées particulières. Par suite, lorsqu'un inconnu se présente à nous, ses premières apparitions ont toutes chances de nous mettre en mesure de prévoir la catégorie à laquelle il appartient et les attributs qu'il possède, son "identité sociale" [...].

Nous appuyant alors sur ces anticipations, nous les transformons en attentes normatives, en exigences présentées à bon droit. $^{40}$

Si l'on s'attache avec précision au texte de Goffman, quelle est la situation? Simplement, la suivante: un ensemble d'athlètes (les footballeurs), participe à un tournoi international dans lequel ils représentent la nation. Ils en sont donc les "champions", au sens médiéval ou classique du terme.

40 GOFFMAN, Erving Goffman. Stigmate. Les usages sociaux des handicaps. Paris: Les

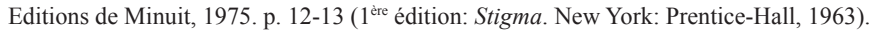


D'abord campium (1080), puis champion (v. 1150), le terme est issu, par le latin médiéval campio (643), du germanique kampjo "combattant dans un duel judiciaire", attesté par l'ancien haut allemand chempfo, chempfjo, le moyen haut allemand kempfe, l'anglo-saxon cempa, et le norrois kappi "combattant". Ces mots viennent du germanique Kamp "lieu du combat", emprunté au latin campus ( $\rightarrow$ champ) par les mercenaires germaniques. Le mot conserve sa valeur étymologique désignant celui qui combat en champ clos pour défendre une cause, et par extension (1552) celui qui combat pour une cause.

(Dictionnaire Historique de la Langue Française, tome 1, p. 695).

Or, la cause à laquelle s'identifie à eux le peuple par divers moyens (le maillot bleu, le coq), c'est bien sûr la nation: la France. Il y a donc ici, vis-à-vis de ces footballeurs, des attentes extrêmement élevées en matière de résultats sportifs, mais aussi et surtout en matière de comportements sur le terrain et hors du terrain. Il y a donc bien des attentes normatives, et des exigences liées à celles-ci. Mais reprenons le cours de la pensée de Goffman:

D'ordinaire, nous n'avons pas conscience d'avoir formulé de telles exigences, ni conscience de leur nature, tant que la satisfaction n'en est pas activement mise en question..$^{41}$ Mais, si elle l'est, c'est alors que nous risquons de nous apercevoir que nous n'avons cessé de poser certaines hypothèses quant à ce que devrait être l'individu qui nous fait face. ${ }^{42}$

Ainsi, il est clair que les commentaires exprimés à l'occasion de ces événements, et par ce moyen les sentiments profonds du peuple vis-à-vis de "ses" footballeurs, a bien montré l'insatisfaction de celui-là à l'encontre de ceux-ci. Et Goffman va plus loin en apportant la réponse au comportement jugé affligeant par la presse écrite, les médias, le personnel politique, les commentateurs sportifs, les journalistes, jusqu'à certains intellectuels, et le peuple en son entier, des joueurs, de leur capitaine et du sélectionneur:

41 C'est moi qui souligne.

42 Erving Goffman, op. cit., p. 12-13. 
[...], il arrive que nous percevions la réaction de défense qu'a l'individu stigmatisé à l'égard de sa situation comme étant l'expression directe de sa déficience, ${ }^{43}$ et qu'alors nous considérons à la fois la déficience et la réaction comme le juste salaire de quelque chose [...], ce qui par suite, justifie la façon dont nous le traitons". ${ }^{44}$

Ainsi, la maladresse avec laquelle les protagonistes de Knysna se sont défendus face aux jugements de valeurs (Max Weber) portés sur eux ont tout simplement renforcé et justifié les impressions initiales: défense d'un banni indéfendable (Anelka), rédaction d'un communiqué, lecture de ce communiqué par le sélectionneur, "grève" de l'entraînement, ultime défaite contre l'Afrique du Sud, jusqu'au refus de Domenech de serrer la main de l'entraîneur brésilien de l'équipe d'Afrique du Sud, Carlos Alberto Parreira, ${ }^{45}$ ce qui ne sied pas du tout à l'image de politesse et de savoir vivre français. ${ }^{46}$ Tout vient alors corroborer et renforcer les sentiments initiaux de déshonneur et d'indignité de cette équipe de France.

[Le problème du stigmate] apparaît dès lors que, de toutes parts, on s'attend plus ou moins à ce que les membres d'une catégorie donnée ne fassent pas que soutenir une certaine norme, mais en outre l'appliquent. ${ }^{47}$

Or, personne dans le cas de l'équipe de France en Afrique du Sud, n'a eu la capacité d'appliquer la norme qui lui était imposée: combattre pour la cause, défendre l'honneur sportif de la nation France, d'où l'ensemble des sentiments qui se sont fait jour et qui, en creux, ou en négatif - pour prendre une métaphore de technique photographique -, indique l'investissement affectif et émotionnel des Français à son égard.

43 C'est moi qui souligne.

44 Erving Goffman, op. cit., p. 16.

45 Entraîneur de l'équipe du Brésil championne du monde en 1994 (Etats-Unis).

46 Cf. supra, note $\mathrm{n}^{\circ} 16$.

47 Erving Goffman, op. cit., p. 16-17. 


\section{Conclusion: les sentiments comme institution}

Ce qui apparaît à l'issue de ce regard porté sur les événements survenus à l'équipe de France en Afrique du Sud, c'est bien, à la suite des remarques initiées par Erving Goffman, que les sentiments qui ont émergé dans la presse et qui exprimaient largement l'ensemble de l'opinion française ont mis en évidence, comme cela a été signalé supra, le ressentiment ${ }^{48}$ vis-à-vis de l'équipe nationale dont les membres ne se sont pas comportés comme on l'attendait: ni sur le terrain, ni en dehors. On s'est retrouvé dans un "dispositif pathétique" 49 qui a fait émerger sentiments et passions: colère, détresse, sentiment d'être outragé devant le monde entier. L'équipe de France a ouvert une "blessure" ${ }^{50}$ dans le cœur des Français, ce qui a conduit à l'expression de ces passions. D'une certaine manière, l'identité nationale s'en est trouvée questionnée, le rapport au monde établi à travers l'équipe de football a été ébranlé, les conceptions morales qui président à ce que doit être une équipe nationale, ont été inquiétées dans leur intégrité..$^{51}$

Tout un ensemble de déclarations de personnalités, en particulier des hommes et femmes politiques, illustrent cette perte de repères à laquelle ont été confrontés les supporteurs et la plupart des Français en ce mois de juin 2010:

Il règne en équipe de France un climat qu'au fond Nicolas Sarkozy a exalté: c'est l'individualisme, l'égoïsme, le chacun pour soi, et la seule échelle de valeur de la réussite humaine, c'est le chèque touché en fin de mois (Jérôme Cahuzac, député PS, Le Dauphiné libéré, supplément Sport, 21 juin 2010, p. 2).

Ils sont devenus les contre-exemples qui montrent le visage de l'irresponsabilité, du narcissisme, des dérives de l'argent, du langage vulgaire ${ }^{52}$ (Christian Vanneste, député UMP, Libération, 23 juin 2010, p. 3).

48 Luiz Carlos Ribeiro, art. cit., p. 106.

49 Mariella Pandolfi \& Vincent Crapanzano, art. cit., p. 10.

50 Mariella Pandolfi \& Vincent Crapanzano, art. cit., p. 10.

51 Mariella Pandolfi \& Vincent Crapanzano, art. cit., p. 10.

52 L'insulte qu'aurait proféré Anelka à l'adresse du sélectionneur ("Va te faire enculer, sale fils de pute!", rapportée par L'Équipe, 19 juin 2010), répond d'une certaine manière aux paroles du président Sarkozy, quelques mois auparavant, à l'adresse d'un manifestant qui l'importunait ('Casse-toi, pauv' con!'). 
C'est une défaite sportive et morale [...]. L'équipe de France est une équipe de traiders, où on ne se rend pas compte de ce qui est autorisé et de ce qui ne l'est pas (François Hollande, député PS, Aujourd'hui en France, 23 juin 2010, p. 4).

Quand un joueur est sélectionné, il joue pour la France et au nom de la France. Il doit rendre à son pays ce que son pays lui a donné. Il y a un certain nombre de devoirs à respecter (Jean-François Copé, député UMP, Le Monde, 24 juin 2010, p. 28).

Pour aller plus loin dans l'analyse, et revenir à la question des institutions - et l'équipe de France de football doit être considérée comme telle étant donné ce qu'elle représente pour la nation et l'intérêt que celle-ci lui porte, toutes composantes confondues - une longue citation est proposée qui invite à réfléchir d'une autre manière aux analyses proposées par Erving Goffman, et qui d'une certaine manière les complète:

Ce domaine [les institutions] comprend toutes les activités régies par des anticipations stables et réciproques. Dans la mesure où son rôle lui confère un pouvoir, l'acteur peut chercher à profiter pour son propre compte es avantages que lui réserve son rôle; ou bien, prenant comme référence non pas lui-même, et ses propres intérêts, caprices et humeurs, mais les responsabilités qu'il assume dans l'exécution d'une tâche et au point de vue de ses partenaires. Lesdits partenaires ne sont pas nécessairement ses égaux; il peut être plus fort, plus compétent qu'eux. Pourtant, il existe certaines situations, celles précisément qui seront dites institutionnalisables, où le rôle ne peut pas être joué si l'acteur n'accepte pas de "se mettre à la place d'autrui". Tout rapport paisible et régulier cesserait, si une des parties découvrait qu'elle est "manipulée", abusée ou "exploitée" par l'autre. ${ }^{53}$

N'est-ce pas ce qui s'est passé avec l'équipe de France? Et la réaction des supporteurs et des médias a été d'autant plus violente qu'ils ont

53 BOUDON, Raymond; BOURRICAUD, François. Dictionnaire Critique de la Sociologie. Paris: Presses Universitaires de France, 1982. p. 312. 
découvert, avec effarement, qu'ils étaient manipulés, abusés, exploités, que le maillot bleu ne représentait plus rien aux yeux des joueurs malgré leurs déclarations, et que ce n'était effectivement qu'arrogance, individualisme et chasse aux sorcières qui prévalaient. ${ }^{54} \mathrm{~A}$ aucun moment, les acteurs de cette tragi-comédie n'ont eu la capacité de se décentrer: "Plus que jamais, [l'équipe nationale] est coupée de l'ensemble des Français qui ne se reconnaissent pas dans ces joueurs qui affichent leur manque d'éducation, leur jalousie, leur arrogance dans le sillage du sélectionneur" (Aujourd'hui en France, 20 juin 2010, p. 2).

Sur l'autocar qui, en Afrique du Sud, transportait les joueurs entre leur hôtel, le terrain d'entraînement, l'aéroport, etc., avaient été écrits les mots suivants: "Tous ensemble vers un nouveau rêve bleu"...

Recebido em julho de 2012. Aprovado em agosto de 2012.

54 'Notre problème n'est pas Anelka mais le traître qui est parmi nous. Il faut éliminer ce traître du groupe. Le groupe n'est pas sain" déclare Patrice Evra, le capitaine de l'équipe de France ( $L e$ Figaro, 21 juin 2010, p. 14); et Franck Ribéry, sur l'antenne de la chaîne de télévision TF1: "Le groupe a explosé. Un traitre a sorti trop de trucs, on aura un soulagement de savoir qui c'est" (Le Figaro, 21 juin 2010, p. 14). 